\title{
Molecular Dynamics Simulations of Two-Dimensional Clusters of Charges
}

\author{
Simara S. de Moraes ${ }^{1,2}$, Débora Coimbra ${ }^{1}$, J. Ricardo de Sousa ${ }^{1}$, and Hidemberg Ordozgoith da Frota ${ }^{1}$ \\ ${ }^{1}$ Departamento de Física, Universidade Federal do Amazonas, 69077000, Manaus, AM, Brazil \\ ${ }^{2}$ Centro Universitário Luterano de Manaus, Universidade Luterana do Brasil, 69077730, Manaus, AM, Brazil
}

Received on 6 January, 2004

\begin{abstract}
We study structural and spectral properties of finite classical systems of $N$ two-dimensional charged particles, confined by a parabolic potential $\propto r^{n}$, and interacting via inverse power-law potentials $\propto 1 / r^{n^{\prime}}$. Molecular dynamics simulations are performed for different cluster sizes $(N=30$ to 230$)$ and $n, n^{\prime}$ values $1,2,3$ and 10 . We also analyze the phase transition from a ring-like configuration to a Wigner structure as a function of parameter $n^{\prime}$ and anisotropy. We compare our results with Monte Carlo simulations of Bedanov and Peeters, obtaining good agreement. In addition, we determine the Voronoi structure of the cluster. Our work complements that of Cândido, Rino and Studart, who analyzed confinement in a screened parabolic potential.
\end{abstract}

\section{Introduction}

The two-dimensional classical atom is a model system in which $N$ equally charged classical particles move in a plane, and are kept together by a parabolic potential. For each value of $N$ we have at least one stable configuration. Examples of 2D Coulomb clusters are electrons on the surface of liquid helium [1] and electrons in quantum dots [2]. After the discovery of Wigner crystallization [3] of electrons on the surface of liquid helium [4] (a two-dimensional system), there has been considerable theoretical and experimental progress in the study of the mesoscopic system consisting of a finite number of charged particles, which are laterally confined by a parabolic potential and interact each other through a potential. This system is the classical analog of the well known quantum dot problem, if the interaction potential is the Coulomb one. In a finite system there is a competition between the bulk triangular lattice and the circular confinement potential that tries to force the particles into a ringlike configuration. These quantum dots are atomlike structures that have interesting optical properties and may be of interest for single-electron devices [5]. These systems and their configurations have been observed experimentally [6], and are important in solid-state physics [10], plasma physics [7] as well as in atomic physics [8]. Bolton and Rössler [9] implemented Monte Carlo simulation of isotropic parabolic confinement, to investigate the minimum energies and spatial distributions for small numbers (1-40) of particles.

Ringlike configurations in two-dimensional clusters were systematically investigated by Bedanov and Peeters [11] using Monte Carlo method and a Mendeleev-type of table for these classical atomlike structures was constructed, for up to 230 particles. The spectral properties of the ground-state configurations were studied by Schweigert and Peeters [12] and generalized to screened Coulomb interac- tions by Cândido et al. [13] and to logarithmic interactions [14, 15, 16]. In particular, Partoens and Peeters [15] compared the values obtained using Monte Carlo simulation with the Thomson model for a two-dimensional system constituted for $N$ classical particles confined by potentials $\propto r^{n}$.

Recently, Kong et al. [5] pointed out the importance and treatment of metastable states in numerical simulations. They also investigated the stability of the ground-state configurations against the functional form of the confinement potential and the exact form of the interparticle interaction potential.

In this work, we study structural and spectral properties of finite classical systems of $N$ charged two-dimensional particles, confined by a parabolic potential interacting by inverse power potentials. The molecular dynamics simulation was performed to simulate clusters containing about 230 particles.

This paper is organized as follows. In Section 2, we described the model and introduced the dimensionless formulation. In Section 3, we presented our results, calculating the energy and the space arrangement of particles. Finally, our conclusions were performed out in last section.

\section{Model and Method}

We study a $2 \mathrm{D}$ system consisting of a finite number $N$ identical classical particles, with dielectric constant $\epsilon$, confined by means of a potential of the form

$$
V_{c}=\frac{1}{2} m \omega^{2} \sum_{i=1}^{N}\left(x_{i}^{2}+\alpha y_{i}^{2}\right)^{n / 2},
$$

where $m$ is the particle mass, $\left(x_{i}, y_{i}\right)$ are the coordinates of the particles, whose vectorial position is $\mathbf{r}_{\mathbf{i}}, \omega$ is the fre- 
quency of the confinement potential, $\alpha$ is the anisotropic parameter in the direction of the axis $y$. The particles interact through the potential

$$
V_{i n t}=\frac{q^{2}}{\epsilon} \sum_{i>j}^{N} \frac{1}{\left|\mathbf{r}_{\mathbf{i}}-\mathbf{r}_{\mathbf{j}}\right|^{n \prime}}
$$

or

$$
V_{i n t}^{\prime}=-\frac{q^{2}}{\epsilon} \sum_{i>j}^{N} \ln \left|\mathbf{r}_{\mathbf{i}}-\mathbf{r}_{\mathbf{j}}\right|,
$$

where $q$ is the charge of each particle and $\epsilon$ is dielectric constant of the medium.

The total potential energy is

$$
V=\frac{1}{2} m \omega^{2} \sum_{i=1}^{N}\left(x_{i}^{2}+\alpha y_{i}^{2}\right)^{n / 2}+\frac{q^{2}}{\epsilon} \sum_{i>j}^{N} \frac{1}{\left|\mathbf{r}_{\mathbf{i}}-\mathbf{r}_{\mathbf{j}}\right|^{n \prime}} .
$$

Eq. (4) can be written in a dimensionless form [15], expressing the coordinates, the energy, a temperature and the force in terms of the units $r_{0}, E_{0}, T_{0}$ e $F_{0}$, respectively, given by

$$
\begin{aligned}
& r_{0}=\left[\frac{q^{2}}{\gamma \epsilon}\right]^{1 /\left(n+n^{\prime}\right)} \\
& E_{0}=\left[\frac{q^{2}}{\epsilon}\right]^{n /\left(n+n^{\prime}\right)} \gamma^{n^{\prime} /\left(n+n^{\prime}\right)} \\
& F_{0}=\left[\frac{q^{2}}{\epsilon}\right]^{(n+1) /\left(n+n^{\prime}\right)} \gamma^{\left(n^{\prime}+1\right) /\left(n+n^{\prime}\right)} \\
& T_{0}=\left[\frac{q^{2}}{\epsilon}\right]^{n /\left(n+n^{\prime}\right)} \gamma^{n^{\prime} /\left(n+n^{\prime}\right)} k_{B}^{-1},
\end{aligned}
$$

where $\gamma=\frac{1}{2} m \omega^{2}$. In terms of these dimensionless units, the Hamiltonian of Eq. (4) becomes

$$
V=\sum_{i=1}^{N}\left(x_{i}^{2}+\alpha y_{i}^{2}\right)^{n / 2}+\sum_{i>j}^{N} \frac{1}{\left|\mathbf{r}_{\mathbf{i}}-\mathbf{r}_{\mathbf{j}}\right|^{n \prime}}
$$

or

$$
V=\sum_{i=1}^{N} r_{i}^{2}-\sum_{i>j}^{N} \ln \left|\mathbf{r}_{\mathbf{i}}-\mathbf{r}_{\mathbf{j}}\right|
$$

This potential has also been studied for Partoens and Peeters [15], using Monte Carlo methods, as previously mentioned.

Thus, the energy involves the following parameters: the power $n$ of the confinement potential, the power $n^{\prime}$ of the interaction potential, number $N$ of particles and $\alpha$. The Hamiltonian of Eq. (9) was studied by Partoens and Peeters [15], who generalized the Thomson model to investigate the effect of different interaction potential between particles on the ground-state configurations. They compared their results with numerical Monte Carlo simulations. For $n=2$ and $n^{\prime}=1$, the $N$ particles system is under a Coulomb potential and are confined by a parabolic potential. This case was studied by Bedanov and Peeters [11] using the Monte Carlo method, with $\alpha=1$. In agreement with Ref. [11], the typical values given by the Eqs. (5) - (8) are
1. For electrons above liquid helium $(q=-e, \epsilon=1$, $m=m_{e}$ ) with confinament potential such that $\hbar \omega=$ $0.1 \mathrm{meV}: r_{0}=2800 \AA, E_{0}=5.1 \mathrm{meV}, T_{0}=60 \mathrm{~K} \mathrm{e}$ $F_{0}=29.4 \times 10^{-16} \mathrm{~N}$.

2. For electrons in a GaAs heterostructure, with a typical energy of quantum dot confinement $\hbar \omega=1$ $\mathrm{meV}: r_{0}=630 \AA, E_{0}=1.7 \mathrm{meV}, T_{0}=20 \mathrm{~K} \mathrm{e}$ $F_{0}=6.32 \times 10^{-16} \mathrm{~N}$.

The Molecular Dynamics Simulation (MD) is a method to solve classical Newton's equations of movement of a particle system. Early on, Alder and Wainwright [17] applied it to study the hard sphere fluid. Afterwards, many authors analyzed Lennard-Jones fluids using this method [18, 19]. In this work, we use the predictor corrector algorithm to solve the equations of motion for the Hamiltonian of Eq. (9). For references on basic MD implementation see [20].

\section{Results}

In Table I and II, we presented the minimum state configuration and energy $E / N$ for parabolic confinement and a logarithmic interaction between particles, as well as an interaction proportional to $\left|\mathbf{r}_{\mathbf{i}}-\mathbf{r}_{\mathbf{j}}\right|^{-1}, \quad\left|\mathbf{r}_{\mathbf{i}}-\mathbf{r}_{\mathbf{j}}\right|^{-2}$ and $\left|\mathbf{r}_{\mathbf{i}}-\mathbf{r}_{\mathbf{j}}\right|^{-3}$. The last represents an original contribution of this work. For simplicity, we adopt the notation $r=$ $\left|\mathbf{r}_{\mathbf{i}}-\mathbf{r}_{\mathbf{j}}\right|$. For the logarithmic potential, for $N$ of 2 to 6 the particle arrangement is a ring; for $N=7$ to $N=16$ the particles form two concentric rings; for $N=17$ to $N=33$, three concentric rings. For $N$ between 34 and 51 we obtain four rings. Our results are in a good agreement with those obtained by Partoens and Peeters [15]. Despite this, for more than one ring, our results for the maximum number of particles that are distributed in a certain number of rings in general differ from those of [15]. Perhaps this discrepancy is a consequence of the approach used in the Thomson model. For $N>6$, the system is unstable if we impose only one ring, because the excess charges are placed in the center of the ring. Considering the confinement potential with $n=2$ and $\alpha=1$, and the interaction potential with $n^{\prime}=$ 1, 2 and 3, in Eq. (9), we found for a repulsive Coulomb interaction $\left(n^{\prime}=1\right)$ a single ring for $N$ varying from 2 to 5 ; two rings for $N$ varying from 6 to 15 ; three rings for $N=$ 16 to $N=31$; and four rings for $N=32$ to $N=51$.

We compared our results with those obtained with the Thomson model [15] shown in Table III, observing a good agreement. We also compared our results with obtained with the Monte Carlo method (MC) for Bedanov e Peerters [11]. The same number of rings are found with DM and MC, because both methods have a good precision. The number of charges per ring is also generally in agreement. Only for $N=21,29$ and 51 the rings do not have the same number of charges. For an interaction proportional to the square of the distance and $N$ varying of 2 to 5 we obtained only one ring; for $N$ varying of 6 to 15 two rings; for $N=16$ the $N=29$ three rings; for $N=30$ to 48 four 
TABLE I. Ground-state configurations and energies for parabolic confinement and logarithmic or power-law potentials, proportional to $r$, $r^{2}$ and $r^{3}$ interaction between the particles.

\begin{tabular}{|c|c|c|c|c|c|c|c|c|}
\hline & \multicolumn{2}{|c|}{$\ln r$} & \multicolumn{2}{|l|}{$1 / r$} & \multicolumn{2}{|l|}{$r^{-2}$} & \multicolumn{2}{|l|}{$r^{-3}$} \\
\hline$N$ & $N_{1}, N_{2}$ & . $E / N$ & $N_{1}, N_{2}$ & $E / N$ & $N_{1}, N_{2}$ & $E / N$ & $N_{1}, N_{2}$ & $E / N$ \\
\hline 2 & 2 & 0.250000 & 2 & 0.750000 & 2 & 0.707106 & 2 & 0.646023 \\
\hline 3 & 3 & 0.297267 & 3 & 1.310371 & 3 & 1.154701 & 3 & 1.033940 \\
\hline 4 & 4 & 0.272614 & 4 & 1.835445 & 4 & 1.581138 & 4 & 1.380219 \\
\hline 5 & 5 & 0.195281 & 5 & 2.338445 & 5 & 2.000000 & 5 & 1.757129 \\
\hline 6 & 6 & 0.075191 & 1,5 & 2.804556 & 1,5 & 2.357023 & 1,5 & 2.048295 \\
\hline 7 & 1,6 & -0.107321 & 1,6 & 3.238974 & 1,6 & 2.687841 & 1,6 & 2.325912 \\
\hline 8 & 1,7 & -0.314343 & 1,7 & 3.668904 & 1,7 & 3.031089 & 1,7 & 2.635424 \\
\hline 9 & 1,8 & -0.546056 & 2,7 & 4.088112 & 2,7 & 3.359067 & 2,7 & 2.923727 \\
\hline 10 & 2,8 & -0.810041 & 2,8 & 4.484942 & 3,7 & 3.664240 & 3,7 & 3.190119 \\
\hline 11 & 3,8 & -1.099393 & 3,8 & 4.864668 & 3,8 & 3.939216 & 3,8 & 3.419723 \\
\hline 12 & 3,9 & -1.414881 & 3,9 & 5.238936 & 3,9 & 4.222073 & 3,9 & 3.666648 \\
\hline 13 & 4,9 & -1.747393 & 4,9 & 5.601138 & 4,9 & 4.486358 & 4,9 & 3.894935 \\
\hline 14 & 4,10 & -2.101995 & 4,10 & 5.958983 & 5,9 & 4.756484 & 5,9 & 4.135425 \\
\hline 15 & 4,11 & -2.470745 & 5,10 & 6.307582 & 5,10 & 5.011766 & 5,10 & 4.359993 \\
\hline 16 & 5,11 & -2.858568 & $1,5,10$ & 6.649903 & $1,5,10$ & 5.256733 & $1,5,10$ & 4.565577 \\
\hline 17 & $1,5,11$ & -3.259592 & $1,6,10$ & 6.982902 & $1,6,10$ & 5.495558 & $1,6,10$ & 4.772718 \\
\hline 18 & $1,6,11$ & -3.678162 & $1,6,11$ & 7.308140 & $1,6,11$ & 5.728049 & $1,6,11$ & 4.972574 \\
\hline 19 & $1,6,12$ & -4.111396 & $1,6,12$ & 7.631929 & $1,6,12$ & 5.964583 & $1,6,12$ & 5.180093 \\
\hline 20 & $1,6,13$ & -4.555099 & $1,7,12$ & 7.949613 & $1,7,12$ & 6.193368 & $1,7,12$ & 5.388325 \\
\hline 21 & $1,7,13$ & -5.012836 & $2,7,12$ & 8.266450 & $2,7,12$ & 6.422147 & $2,7,12$ & 5.590479 \\
\hline 22 & $1,7,14$ & -5.480377 & $2,8,12$ & 8.574177 & $2,8,12$ & 6.639369 & $2,8,12$ & 5.779691 \\
\hline 23 & $1,8,14$ & -5.959334 & $2,8,13$ & 8.877580 & $3,8,12$ & 6.853577 & $3,8,12$ & 5.968660 \\
\hline 24 & $2,8,14$ & -6.450845 & $3,8,13$ & 9.175904 & $3,9,12$ & 7.062121 & $3,9,12$ & 6.149618 \\
\hline 25 & $3,8,14$ & -6.951869 & $3,9,13$ & 9.470792 & $3,9,13$ & 7.265918 & $3,9,13$ & 6.325612 \\
\hline 26 & $3,9,14$ & -7.463725 & $3,9,14$ & 9.762724 & $4,9,13$ & 7.471314 & $3,9,14$ & 6.508921 \\
\hline
\end{tabular}

TABLE II. Ground-state configurations and energies for parabolic confinement and logarithmic and power-law interactions, proportional to $r, r^{2}$ and $r^{3}$.

\begin{tabular}{|c|c|c|c|c|c|c|c|c|}
\hline & \multicolumn{2}{|l|}{$\ln r$} & \multicolumn{2}{|l|}{$1 / r$} & \multicolumn{2}{|l|}{$r^{-2}$} & \multicolumn{2}{|l|}{$r^{-3}$} \\
\hline$N$ & $N_{1}, N_{2}, \ldots$ & $E / N$ & $N_{1}, N_{2}, \ldots$ & $\bar{E} / N$ & $N_{1}, N_{2}, \ldots$ & & $N_{1}, N_{2}, \ldots$ & \\
\hline 27 & $3,9,15$ & -7.985690 & $4,9,14$ & 10.050860 & $4,9,14$ & 7.671251 & $4,9,14$ & 6.684103 \\
\hline 28 & $4,9,15$ & -8.515336 & $4,10,14$ & 10.335640 & $4,10,14$ & 7.868114 & $4,10,14$ & 6.856542 \\
\hline 29 & $4,10,15$ & -9.054867 & $4,10,15$ & 10.618070 & $5,10,14$ & 8.066881 & $5,10,14$ & 7.035974 \\
\hline 30 & $4,10,16$ & -9.603428 & $5,10,15$ & 10.897317 & $1,5,10,14$ & 8.260623 & $1,6,11,12$ & 7.205670 \\
\hline 31 & $4,10,17$ & -10.158927 & $5,11,15$ & 11.173880 & $1,6,11,13$ & 8.448544 & $1,6,11,13$ & 7.368754 \\
\hline 32 & $4,11,17$ & -10.722904 & $1,5,11,15$ & 11.446579 & $1,6,11,14$ & 8.632648 & $1,6,12,13$ & 7.529165 \\
\hline 33 & $5,11,17$ & -11.295374 & $1,6,11,15$ & 11.715643 & $1,6,11,15$ & 8.813943 & $1,6,12,14$ & 7.687414 \\
\hline 34 & $1,5,11,17$ & -11.874435 & $1,6,12,15$ & 11.982630 & $1,6,12,15$ & 8.993586 & $1,6,12,15$ & 7.844078 \\
\hline 35 & $1,6,11,17$ & -12.461730 & $1,6,12,16$ & 12.246930 & $1,6,12,16$ & 9.173922 & $1,6,12,16$ & 8.003610 \\
\hline 36 & $1,6,12,17$ & -13.056475 & $1,6,12,17$ & 12.510805 & $1,7,12,16$ & 9.356311 & $1,7,12,16$ & 8.172543 \\
\hline 37 & $1,6,12,18$ & -13.658201 & $1,7,12,17$ & 12.771900 & $1,7,13,16$ & 9.534679 & $1,7,13,16$ & 8.332584 \\
\hline 38 & $1,6,12,19$ & -14.265603 & $1,7,13,17$ & 13.030446 & $2,8,13,15$ & 9.710122 & $2,8,13,15$ & 8.485500 \\
\hline 39 & $1,7,13,18$ & -14.879885 & $2,7,13,17$ & 13.287900 & $2,8,13,16$ & 9.882339 & $3,8,13,15$ & 8.638689 \\
\hline 40 & $1,7,13,19$ & -15.501066 & $2,8,13,17$ & 13.541920 & $2,8,14,16$ & 10.052040 & $2,8,14,16$ & 8.787602 \\
\hline 41 & $1,7,14,19$ & -16.12 & $2,8,14,17$ & 13.793970 & $3,9,14,15$ & 10.220142 & $3,9,14,15$ & 8.933968 \\
\hline 42 & $2,7,14,19$ & -16.759985 & $3,8,14,17$ & 14.043960 & $3,9,14,16$ & 10.385550 & $3,9,14,16$ & 9.081480 \\
\hline 43 & $2,8,14,19$ & -17.399222 & $3,9,14,17$ & 14.291540 & $3,9,14,17$ & 10.550290 & $3,9,15,16$ & 9.227055 \\
\hline 44 & $2,8,14,20$ & -18.044561 & $3,9,14,18$ & 14.537528 & $3,9,14,18$ & 10.715280 & $4,10,15,15$ & 9.374854 \\
\hline 45 & $3,8,14,20$ & -18.694880 & $3,9,15,18$ & 14.781877 & $3,9,15,18$ & 10.876680 & $4,10,15,16$ & 9.516908 \\
\hline 46 & $3,9,14,20$ & -19.3 & $4,9,15,18$ & 15.025600 & $4,10,15,17$ & 11.037140 & $4,10,15,17$ & 9.660061 \\
\hline 47 & $3,9,15,20$ & -20.0 & $4,10,15,18$ & 15.266426 & $4,10,15,18$ & 11.197190 & $4,10,16,17$ & 9.803026 \\
\hline 48 & $3,9,15,21$ & -20.680321 & $4,10,15,19$ & 15.505700 & $5,10,15,18$ & 11.356707 & $4,10,16,18$ & 9.943527 \\
\hline 49 & $3,9,15,22$ & -21.352036 & $4,10,16,19$ & 15.743890 & $1,5,11,16,16$ & 11.512232 & $5,11,16,17$ & 10.086670 \\
\hline 50 & $4,10,15,22$ & -22.029103 & $4,11,16,19$ & 15.981000 & $1,5,11,16,17$ & 11.667480 & $1,6,11,16,16$ & 10.218030 \\
\hline 51 & $4,10,16,21$ & -22.711481 & $5,11,16,19$ & 16.215900 & $1,6,11,16,17$ & 11.819870 & $1,6,11,16,17$ & 10.356500 \\
\hline
\end{tabular}


TABLE III. Comparison between results obtained from molecular dynamics and from the Thomson model.

\begin{tabular}{|c|c|c|c|c|c|c|c|c|}
\hline & \multicolumn{2}{|l|}{$r_{i}$} & \multicolumn{2}{|l|}{$\overline{r_{i}^{2}}$} & \multicolumn{2}{|l|}{$\overline{r_{i}^{3}}$} & \multicolumn{2}{|l|}{$r_{i}^{10}$} \\
\hline$N$ & MD & TM & MD & TM & MD & TM & MD & TM \\
\hline 3 & 3 & 3 & 3 & 3 & 3 & 3 & 3 & 3 \\
\hline 4 & 1,3 & 1,3 & 4 & 4 & 4 & 4 & 4 & 4 \\
\hline 5 & 1,4 & 1,4 & 5 & 5 & 5 & 5 & 5 & 5 \\
\hline 6 & 1,5 & 1,5 & 1,5 & 1,5 & 6 & 6 & 6 & 6 \\
\hline 7 & 1,6 & 1,6 & 1,6 & 1,6 & 1,6 & 7 & 7 & 7 \\
\hline 8 & 1,7 & 2,6 & 1,7 & 1,7 & 1,7 & 1,7 & 8 & 8 \\
\hline 9 & 1,8 & 2,7 & 2,7 & 2,7 & 1,8 & 1,8 & 9 & 9 \\
\hline 10 & $1,4,5$ & 2,9 & 2,8 & 2,8 & 2,8 & 1,9 & 1,9 & 10 \\
\hline 11 & $1,3,7$ & 3,8 & 3,8 & 2,9 & 2,9 & 2,9 & 1,10 & 11 \\
\hline 12 & $1,4,7$ & 3,9 & 3,9 & 3,9 & 3,9 & 2,10 & 1,11 & 12 \\
\hline 13 & $1,4,8$ & $1,3,9$ & 4,9 & 4,9 & 3,10 & 3,10 & 1,12 & 13 \\
\hline 14 & $1,5,8$ & $1,4,9$ & 4,10 & 4,10 & 4,10 & 4,10 & 2,12 & 14 \\
\hline 15 & $1,5,9$ & $1,4,10$ & 5,10 & 5,10 & 4,11 & 4,11 & 3,12 & 15 \\
\hline 16 & $1,5,10$ & $1,5,10$ & $1,5,10$ & 5,11 & 5,11 & 5,11 & 3,13 & 16 \\
\hline 17 & $1,6,10$ & $1,5,11$ & $1,6,10$ & $1,5,11$ & 5,12 & 5,12 & 3,14 & 17 \\
\hline 18 & $1,7,10$ & $1,6,11$ & $1,6,11$ & $1,6,11$ & 6,12 & 6,12 & 4,14 & 1,17 \\
\hline 19 & $1,8,10$ & $2,6,11$ & $1,6,12$ & $1,6,12$ & $1,6,12$ & 7,12 & 4,15 & 2,17 \\
\hline 20 & $1,8,11$ & $2,7,11$ & $1,7,12$ & $1,7,12$ & $1,6,13$ & 7,13 & 4,16 & 3,17 \\
\hline 21 & $1,4,8,8$ & $2,7,12$ & $2,7,12$ & $2,7,12$ & $1,7,13$ & $1,7,13$ & 5,16 & 4,17 \\
\hline 22 & $1,4,8,9$ & $2,8,12$ & $2,8,12$ & $2,7,13$ & $1,7,14$ & $1,8,13$ & 5,17 & 4,18 \\
\hline 23 & $1,4,8,10$ & $3,8,12$ & $2,8,13$ & $2,8,13$ & $1,8,14$ & $1,9,13$ & $1,5,17$ & 5,18 \\
\hline 24 & $1,4,8,11$ & $3,8,13$ & $3,8,13$ & $2,9,13$ & $2,8,14$ & $1,9,14$ & $1,6,17$ & 6,18 \\
\hline
\end{tabular}

rings and for $N=49$ to 51 five rings. We also observed good agreement with the results of Partoens and Peeters [15] for systems with less than 18 particles. The discrepancies for larger numbers of particles occur because to stabilize the system in Thomson model, the internal particles are considered located in the external orbit center. Koulakov and Shklovskii [21] showed for $N$ large, considering Coulomb interaction, the energy is proportional to the particle number as $E \sim N^{5 / 3}$. This behavior is plotted in Fig. 1 for all potentials previously mentioned.

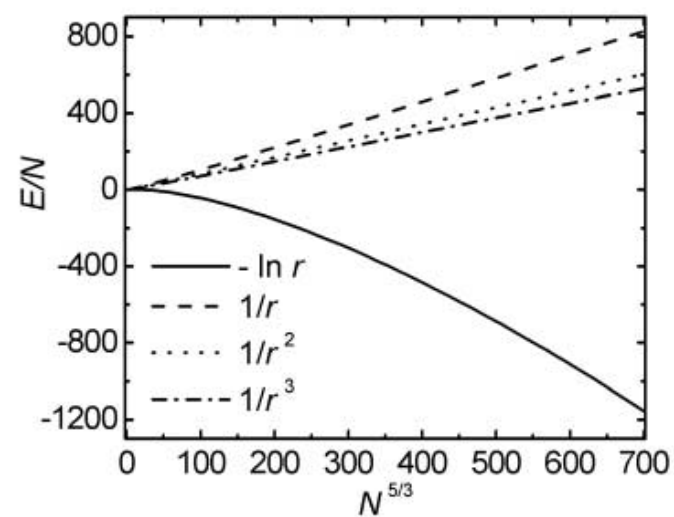

Figure 1. Energy $E$ as a function of particle number $N$, for isotropic parabolic confinement potential and interaction potentials proportional to: $-\ln r$ and $r^{-n^{\prime}}$, with $n^{\prime}=1,2$ and 3 .

Fixing the kind of interaction (Coulomb), we present in Table IV the distribution of charges and respective energy of the ground-state to several confinement potential. In Fig. 2 we show the configurations, with $N=13$ and $n$ assuming the values of $n=1,2,3,7,10$ and 11 in confinement potential. As $n$ increases the particles shift to external rings, but the diameter decreases.
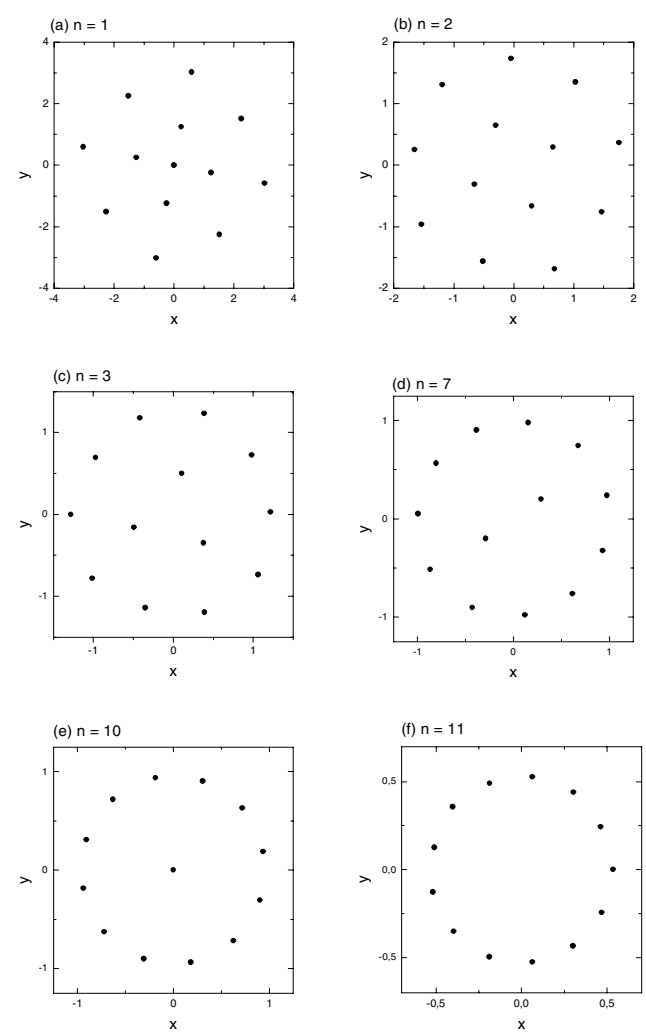

Figure 2. Evolution of the ground-state configurations for a cluster of $N=13$ particles for different values of the confinement potential parameter $n=1,2,3,7,10$ and 11 . 
TABLE IV. Comparison between ground-state configurations and energies.

\begin{tabular}{llllrlrlr}
\hline \hline$N$ & $r_{i}$ & $E / N$ & $r_{i}^{2}$ & $E / N$ & $r_{i}^{3}$ & $E / N$ & $r_{i}^{10}$ & $E / N$ \\
\hline 3 & 3 & 0.519611 & 3 & 1.310371 & 3 & 1.162247 & 3 & 0.823054 \\
4 & 1,3 & 1.883897 & 4 & 1.835445 & 4 & 1.698006 & 4 & 1.303145 \\
5 & 1,4 & 2.238348 & 5 & 2.338445 & 5 & 2.229837 & 5 & 1.813126 \\
6 & 1,5 & 2.569255 & 1,5 & 2.804556 & 6 & 2.757943 & 6 & 2.345965 \\
7 & 1,6 & 5.882525 & 1,6 & 3.238974 & 1,6 & 3.279494 & 7 & 2.897092 \\
8 & 1,7 & 1.175864 & 1,7 & 3.668904 & 1,7 & 3.763418 & 8 & 3.463335 \\
9 & 1,8 & 3.439630 & 2,7 & 4.088112 & 1,8 & 4.249335 & 9 & 4.042382 \\
10 & $1,4,5$ & 3.688346 & 2,8 & 4.484942 & 2,8 & 4.733908 & 1,9 & 4.620593 \\
11 & $1,3,7$ & 3.919288 & 3,8 & 4.864668 & 2,9 & 5.197296 & 1,10 & 5.191859 \\
12 & $1,4,7$ & 4.138402 & 3,9 & 5.238936 & 3,9 & 5.650131 & 1,11 & 5.774198 \\
13 & $1,4,8$ & 4.348932 & 4,9 & 5.601138 & 3,10 & 6.097881 & 1,12 & 6.366374 \\
14 & $1,5,8$ & 4.553242 & 4,10 & 5.958983 & 4,10 & 6.536894 & 2,12 & 6.964201 \\
15 & $1,5,9$ & 4.750637 & 5,10 & 6.307582 & 4,11 & 6.972192 & 3,12 & 7.568404 \\
16 & $1,5,10$ & 4.943978 & $1,5,10$ & 6.649903 & 5,11 & 7.401072 & 3,13 & 8.143586 \\
17 & $1,6,10$ & 5.130189 & $1,6,10$ & 6.982902 & 5,12 & 7.826542 & 3,14 & 8.727266 \\
18 & $1,7,10$ & 5.312896 & $1,6,11$ & 7.308140 & 6,12 & 8.247335 & 4,14 & 9.323835 \\
19 & $1,8,10$ & 5.489154 & $1,6,12$ & 7.631929 & $1,6,12$ & 8.660476 & 4,15 & 9.906046 \\
20 & $1,8,11$ & 5.658290 & $1,7,12$ & 7.949613 & $1,6,13$ & 9.069334 & 4,16 & 10.495631 \\
21 & $1,4,8,8$ & 5.823420 & $2,7,12$ & 8.266450 & $1,7,13$ & 9.471366 & 5,16 & 11.087036 \\
22 & $1,4,8,9$ & 5.985276 & $2,8,12$ & 8.574177 & $1,7,14$ & 9.873136 & 5,17 & 11.675386 \\
23 & $1,4,8,10$ & 6.142535 & $2,8,13$ & 8.877580 & $1,8,13$ & 10.271599 & $1,5,17$ & 12.273630 \\
24 & $1,4,8,11$ & 6.295999 & $3,8,13$ & 9.175904 & $2,8,14$ & 10.668143 & $1,6,17$ & 12.860516 \\
\hline \hline
\end{tabular}
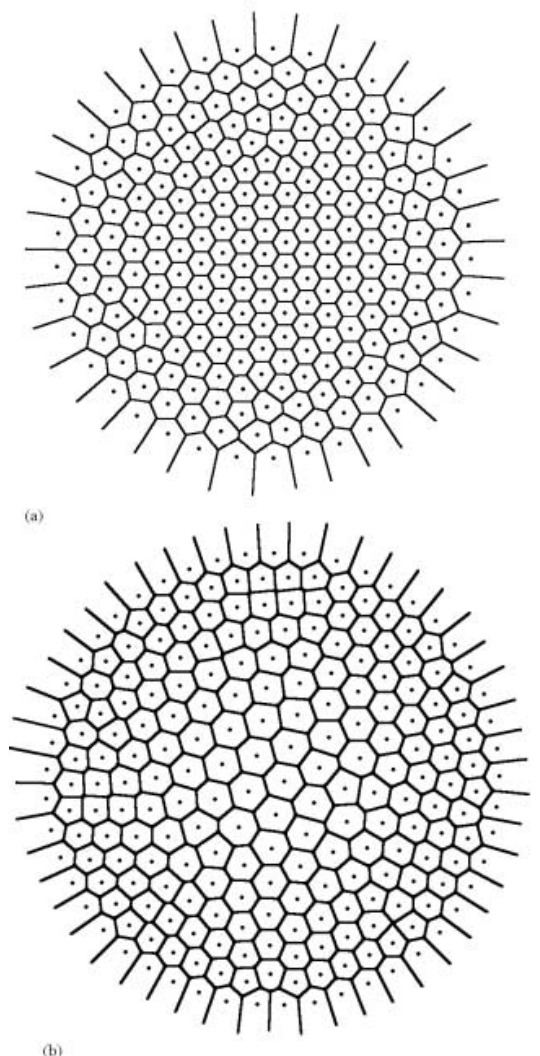

Figure 3. Ground-state configurations and Voronoi structures for 230 particles, confinement potential proportional (a) $r^{2}$ and (b) $r^{5}$, and Coulomb interaction.
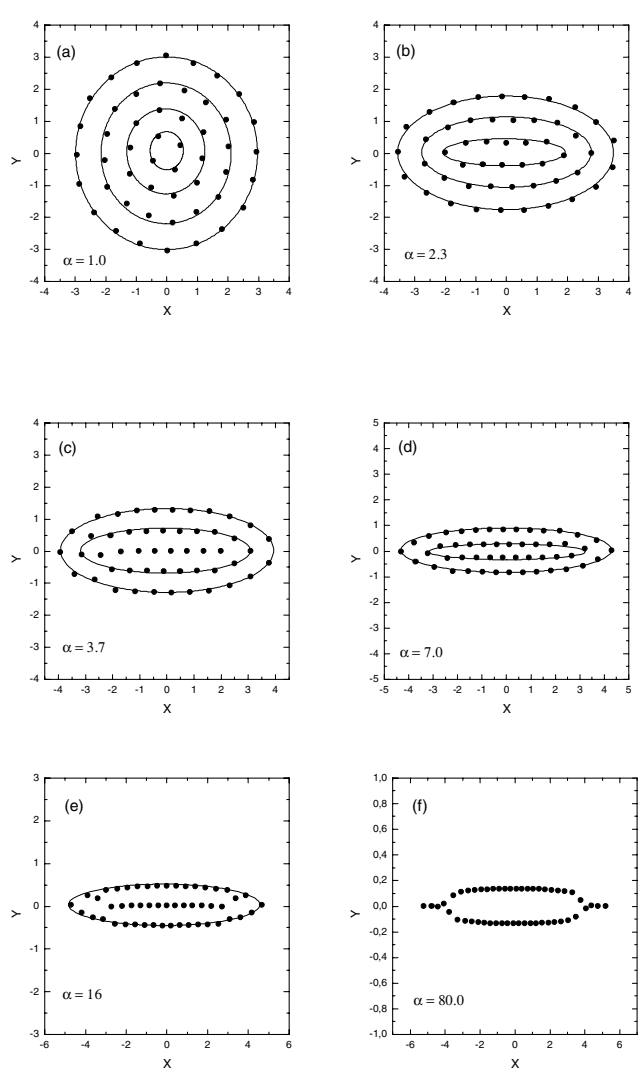

Figure 4. Configuration of $N=50$ particles for several values of the anisotropy parameter $\alpha$ : (a) 1.0, (b) 2.3, (c) 3.7, (d) 7.0, (e) 16.0 and (f) 80.0 . 
In Fig. 3, we presented the Voronoi structure of the particles for the parabolic confinement potential and the Coulomb interaction among the particles with $n=2$ (a) and $n=5$ (b). We observed that the internal rings form a Wigner structure with hexagonal symmetry for $n=2$. However, for $n=5$, the particles tend to migrate to the external layers, breaking the hexagonal symmetry in the central area of the distribution. We also analyzed the anisotropy in the $y$ direction, as presented in Fig. 4. As $\alpha$ increases, the ring configurations tend to elliptical ones.

\section{Final Remarks}

We present results of numerical simulation (MD) of confined interacting particles, in clusters of 30 to 230 particles. The energies and configurations obtained are in agreement with literature values. The importance of these studies lies in their reduced complexity in two-dimensional systems, in relation to three-dimensional ones.

We find that the particle arrangement is ring-like in the ground state. These configuration vary with anisotropy, becoming increasingly elliptical. The energy could be fitted as $\mathrm{N}^{5 / 3}$. This study highlights the possibility to analyze the energy variation with particle number, extensivity, phase transitions and critical points, taking to account metastable states and field interactions.

\section{References}

[1] P. Leiderer, W. Ebner, and V. B. Shikin, Surf. Sci. 113, 405 (1987).

[2] Nanostructure Physics and Fabrication, edited by M. A. Reed and W. P. Kirk (Academic Press, Boston, 1989).

[3] E. P. Wigner, Phys. Rev. 46, 1002 (1934).

[4] C. C. Grimes and G. Adams, Phys. Rev. Lett. 42, 795 (1979).

[5] M. Kong, B. Partoens and F. M. Peeters, Phys. Rev. E 65, 046602 (2003).

[6] D. J. Wineland and W. M. Itano, Phys. Today 40 (6), 34 (1987).

[7] J. H. Chu and L. I, Phys. Rev. Lett. 72, 4009 (1994); H. Thomas, G. E. Morfill, V. Demmel, J. Goree, B. Feuerbacher, and D. M. Mohlmann, Phys. Rev. Lett. 73, 652 (1994); W.-T. Juan, Z.-H. Huang, J.-W. Hsu, Y.-J. Lai, and L. I, Phys. Rev. E 58, 6947 (1998).

[8] S. L. Gilbert, J. J. Bollinger, and D. J. Wineland, Phys. Rev. Lett. 60, 2022 (1988).

[9] F. Bolton and U. Rössler, Superlatt Microstruct. 13, 139 (1993).

[10] A. Rahman and J. P. Schiffer, in Condensed Matter Theories, edited by P. Vashishta et al.(Plenum Press, New York, 1987), Vol. 2, p. 33; J. Beebe-Wang, N. Elander, and R. Schuch, Phys. Scr. 46, 506 (1992); D. H. E. Dubin and T. M. O'Neil, Phys. Rev. Lett. 60, 511 (1988).

[11] V. M. Bedanov and F. M. Peeters, Phys. Rev. B 49, 2667 (1994).

[12] V. A. Schweigert and F. M. Peeters, Phys. Rev. B 51, 7700 (1995); V. A. Schweigert and F. M. Peeters, Phys. Rev. B 51, 7700 (1998).

[13] L. Cândido, J.-P. Rino, N. Studart, Braz. J. Phys. 27/A, 312 (1997); L. Cândido, J.-P. Rino, N. Studart, J. Phys.: Condens. Matter 10, 11627 (1998).

[14] Y.-J. Lai and L. I, Phys. Rev. E 60, 4743 (1999).

[15] B. Partoens and F. M. Peeters, J. Phys.: Condens. Matter 9, 5383 (1997).

[16] L. J. Campbell and R. M. Ziff, Phys. Rev. B 20, 1886 (1979); P. Cheung, M. F. Choi, and P. M. Hui, Solid State Commun. 103, 357 (1997).

[17] B. J. Alder and T. E. Wainwright, J. Chem. Phys. 27, 1208 (1957); B. J. Alder and T. E. Wainwright, J. Chem. Phys. 31, 459 (1959).

[18] A. Rahman, Phys. Rev. 136 (2A), 405 (1964).

[19] L. Verlet, Phys. Rev. 159, 98 (1964); L. Verlet, Phys. Rev. 165, 201 (1968).

[20] D. Frenkel and B. Smit, Understanding Molecular Simulation (Academic Press, San Diego, 1996); M. P. Allen and D. J. Tildesley, Computer Simulation of Liquids (Claredon Press, Oxford, 1986).

[21] A. A. Koulakov, V. A., and F. M. Peeters, Phys. Rev. B 57, 2352 (1998). 